Manuelle Medizin 2016 $54: 1-3$

DOI 10.1007/s00337-015-0079-2

Online publiziert: 7. Januar 2016

(c) Springer-Verlag Berlin Heidelberg 2015

CrossMark

\author{
H. Tilscher ${ }^{1} \cdot A$. Lechner \\ 1 Öst. Ärztegesellschaft für Manuelle Medizin, Geriatriezentrum am Wienerwald, Wien, Österreich \\ ${ }^{2}$ Wien, Österreich
}

\title{
Wirbelsäulenstörungen: Eine Aufgabe für die Praxis - vom Befund zur Behandlung
}

\section{Jahre (konservative) Orthopädie}

Doch man lernt aus der Geschichte, die Gegenwart besser zu verstehen und aus deren Details Prognosen zu erstellen.

$\mathrm{Zu}$ Beginn meiner Ausbildung zum Orthopäden im Jahre 1965 litten die Patienten an erworbenen oder angeborenen Veränderungen des Skeletts (Rachitis, Skoliosen, Hüftluxationen, Z. n. Kriegsverletzungen), an Operationen gab es die Meniskektomien, Halluxoperationen, die Voss-Hängehüfte, etc. Eine wichtige Rolle spielten die Gipsverbände und die physikalische Therapie.

Der Slogan „die Zeiten ändern sich“ lässt sich auch auf Krankheiten übertragen, die im Sinne der Epidemiologie eine deutliche Dynamik aufweisen: So wurde im vorigen Jahrhundert das Zurückdrängen von Seuchen wie der Pest, der Cholera, der Tuberkulose und der Poliomyelitis beobachtet. Das war der sogenannte erste Übergang. Um etwa 1970 wurde ein vermehrtes Auftreten von „degenerativen und durch Verhalten provozierte Erkrankungen“ beschrieben - der zweite Übergang. Derzeit spricht die Epidemiologie (Temmel, 1999) von der Wiederkehr alter und dem Auftreten neuer Infektionskrankheiten (• Abb. 1).

Hier soll auf die in einem Zeitraum von 50 Jahren selbst beobachtete Entwicklungen von Beschwerdesyndromen

Dieser Beitrag basiert auf einem Vortrag, gehalten auf dem Kongress Die gestörte Wirbelsäule - eine Aufgabe für die Praxis - vom Befund zur Behandlung in Pörtschach am Wörthersee im Juli 2015.

Literatur beim Verfasser des Bewegungsapparates, speziell der Wirbelsäule eingegangen werden, welche nach Wegen suchen ließ, um diesen neuartigen Erkrankungen diagnostisch, therapeutisch und letzten Endes auch präventiv entgegentreten zu können. Die Begegnung mit der deutschen manuellen Medizin und ihren genialen Manualmedizinern, die sich mit dieser Problematik intensiv beschäftigten, sollte sich dabei als unschätzbarer Gewinn erweisen.

Durch die Entwicklung der Anästhesie und durch die steigenden technischen Fortschritte konnte die chirurgische Orthopädie in weite Dimensionen vordringen. Die Hüftendoprothetik ist inzwischen einer der erfolgreichsten medizinischen Eingriffe.

Diese operativen Möglichkeiten werden sich jetzt auch durch die Vereinigung mit der Unfallchirurgie zu einer noch breiteren medizinischen Sparte entwickeln, die vorwiegend pathomorphologische Veränderungen des Bewegungsapparates durch ihre anatomische Rekonstruktion erfolgreich angehen sollen.

Dazu oder nebenbei gibt es die „konservative Orthopädie“, eine Äquivokation, denn die Orthopädie war primär konservativ (•Infobox 1 und 2). Eine ihrer Aufgaben sollte die Behandlung von Beschwerden vor allem der Wirbelsäule sein, die sich - so ist anzunehmen - durch statische, dynamische und psychische Noxen oft in ihrer Komplexität zu Funktionsstörungen entwickeln, bei welchen der Schmerz als Warnsignal nicht durch deutliche pathomorphologische Gegebenheiten erklärt werden kann.
Durch die manuelle Medizin motiviert wurde vor allem dieser Krankheitsgruppe großes Interesse gewidmet und trotz Widerstandes für das Fach „Konservative Orthopädie unter besonderer Berücksichtigung der Manuellen Medizin“ habilitiert.

Die häufig auftretenden therapieresistenten und $\mathrm{zu}$ Rezidiven neigenden Krankheitsbildern waren Anlass, eine Abteilung für konservative Orthopädie und Rehabilitation zu gründen, die erste und einzige ihrer Art in Österreich (- Infobox 3). Der Begriff Rehabilitation und seine Definition als „Wiederein-

Infobox 1 Bilder aus der Vergangenheit - 200 Jahre Orthopädie (Rauschmann M.A. und Thomann K.D. )

Die Orthopädie des 19. Jahrhunderts war von privaten Heilanstalten geprägt, die vor allem den wohlhabenden Bevölkerungsschichten vorbehalten waren. In Deutschland entwickelte sich die moderne Orthopädie aus den Krüppelheilanstalten. Im Vordergrund stand die Behandlung der Rachitis, der Infektionen der Knochen und Gelenke, der Skoliose sowie angeborener Fehlbildungen.

Infobox 2 Orthos paedion (konservative) Orthopädie

"Mittel zu lehren, bei den Kindern die Ungestaltheiten des Körpers zu verhüten und zu verbessern" (Nicolas Andry, 1744) "Majestät, das ist die Kunst, die Krummen gerade und die Lahmen gehend zu machen" (Adolf Lorenz zu Kaiser Franz Josef, 1896) Wo bleibt der Schmerz? 


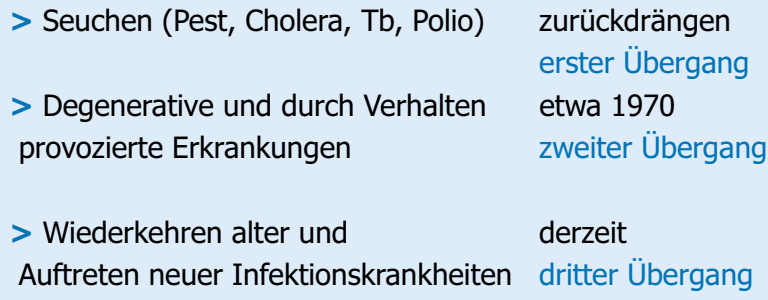

$>$ Wiederkehren alter und

Auftreten neuer Infektionskrankheiten

etwa 1970

zweiter Übergang

derzeit

dritter Übergang

Abb. $1<$ Epidemiologie nach C. Temmel (1999)

\section{Patientenzahl \\ $1974 \quad 1999$ \\ $\begin{array}{ll}n=67 & n=07 \\ w=42(62,7) & w=48(71,7 \%)\end{array}$ \\ Alter \\ \begin{tabular}{lll}
1974 & & 1999 \\
\hline$\varnothing$ & \multicolumn{1}{l}{} & \\
\hline
\end{tabular} \\ Beschwerdedauer \\ 1974

$\emptyset \quad 8,7$ Jahre $\quad \varnothing \quad 11,5$ Jahre \\ Schmerzort: Nacken, Schulter, Arm $\delta$ : $q=1: 3,4$ 1974 \\ $20 \quad(29,8 \%)$ \\ 1999 \\ $31(46,2 \%)$ xx signifikant

Muskuläre Maximalpunkte
$\begin{array}{ll}1974 & 1999 \\ \emptyset 11,06 & \varnothing 18,5-\times \text { signifikant }\end{array}$

Abb. 2 \ Epidemiologie und Übergänge: Zahlen nach 25 Jahren (Abt. für konservative Orthopädie und Schmerztherapie, OSS, Wien, 1971-2002)

gliederung" wurde von uns erweitert in „das Erkennen und die Beeinflussung von krankmachenden Störfaktoren“. Dadurch wird der Patient vom Objekt zum Subjekt. Durch die rasche Entwicklung der chirurgischen Orthopädie wurde die konservative Orthopädie aber in Österreich in den Hintergrund gedrängt, während in Deutschland dennoch orthopädische Kongresse (Baden-Baden, Münster, Erlangen) mit vorwiegend konservativen Themen veranstaltet wurden. Erinnert werden soll auch an die damals zukunftsträchtigen FIMM-Kongresse.

Diese Entwicklungen und die bedeutsamen ökonomischen Konsequenzen der Tätigkeit chirurgischer Opinionleader konnten bewirken, dass bei Durchsicht der Kongressprogramme in Österreich der konservativen Orthopädie relativ wenig Raum gegeben wurde. Doch vor allem für die nachweislich große Zahl der im Wirbelsäulenbereich Erkrankten, bei welchen invasive Maßnahmen nicht angezeigt sind bzw. nur diskutiert werden sollen, ist die qualifizierte medizinische Betreuung dringend notwendig.

Mit etwas differenten Zugängen bieten dies in Österreich neben den physikalischen Medizinern und den niedergelassenen Orthopäden, Rheumatologen, Neurologen, Algesiologen und Psychiatern vor allem aber Allgemeinmediziner an (•Infobox 4).

Zu nennen wäre noch die Kurmedizin, aber in besonderem Maße die Zunahme von nichtärztlichen Heilsanbietern. Unter diesen Umständen wurde die Österreichische Ärztegesellschaft für Manuelle Medizin durch die Bezeichnung ,und Konservative Orthopädie" erweitert und die Kursinhalte neu formiert.

Die 32-jährige Leitung einer $\mathrm{Ab}$ teilung für konservative Orthopädie erlaubte bei Durchsicht der Krankenblätter eine epidemiologische Entwicklung zu erkennen. So zeigte sich eine Zunahme der Beschwerdedauer, des Alters, des Anteils an Frauen und der Häufigkeit von Zervikalsyndromen. Anhand einer bei der Aufnahmeuntersuchung vorgesehenen standardisierten Schmerzpalpation verschiedener Strukturen fand sich eine Vermehrung der Maximalpunkte, die den Schluss auf eine generalisierte gesenkte Schmerzschwelle erlauben (- Abb. 2). Hier muss der Wert der Manipulation relativiert werden, etwa bei den Frauen mit langer Anamnesedauer und psychischen Belastungen, besonders aber bei den Formen orthopädischer Erkrankungen von älteren Menschen, bei welchen eine „Behandlung mit Impuls“ eher nicht vorgesehen ist. In Deutschland beträgt der Anteil an 60-Jährigen $25 \%$, an 80 -Jährigen etwa $5 \%$ (geschätzt 4 Mio.).

Zahllose Schmerzsyndrome verlangen in Abhängigkeit der klinisch zu erhebenden Aktualitätsdiagnose verschiedene therapeutische Zugänge, die sich außer semiinvasiver Verfahren mit der Behandlung der „Peripherie“, d. h.der Schmerzreflektorik, auseinanderzusetzen haben, womit sich eine medizinisch begründete multimodale Behandlung konzipieren lässt, die allerdings wissenschaftlich schwer zu bearbeiten ist. Für die manuelle Medizin bedeutet dies nicht nur, dass die Chirodiagnostik (klinische Untersuchung) für Manualmediziner unverzichtbar ist, sondern auch, dass die Chirotherapie eine der reflextherapeutischen Maßnahmen ist, die in Abhängigkeit vom Befund zum Einsatz kommt.

Bei der Analyse der Krankheitsdynamik muss immer wieder eine multifaktorielle Genese als Ursache, aber nicht unbedingt als Auslöser realisiert werden. Nach der österreichischen Schule für „Manuelle Medizin - Konservative Orthopädie" ist nach diesen Erkenntnissen sofort eine Sekundärprävention angezeigt, die im einzelnen Fall spezifiziert werden müsste, inklusive der Formen der Heilgymnastik, und sich nicht mit der Zuweisung „zur physikalischen Therapie“ erschöpfen dürfte. Einen Beitrag zur Lösung dieser Probleme sollen die von uns jährlich durchgeführten praxisorientierten Kongresse in Pörtschach 


\section{Infobox 3 1971-2002: Abteilung für konservative Orthopädie, Orthopädisches Spital Speising, Wien, (29.500 Patientinnen und Patienten)}

- Multifaktorielle Genese (Rehabilitation),

- Schmerzsyndromatik, multimodale Therapie,

- Chronische Beschwerden - Reiz und Reizbeantwortung - Reflextherapie physikalische Therapie,

- Die Seele ist ein weites Land (Schnitzler)... sie wird immer weiter,

- Sekundärprävention - Tertiärprävention Rehabilitation = Erkennen und Beeinflussen der krankmachenden Störfaktoren.

Infobox 4 Fachrichtungen und ihre therapeutische Strategien

- Orthopädie: Pathomorphologie, operative Rekonstruktion

- Rheumatologie: entzündlicher, degenerativer Rheumatismus

- Neurologie: Kompressionssyndrom

- Algesiologie: Schmerz und seine Phänomene

- Psychiatrie: Somatoforme Angststörungen

- Allgemeinmedizin:?

Infobox 5 Kongresse der Österreichischen Gesellschaft für Manuelle Medizin in Pörtschach am Wörthersee

- Muskeln im Fokus, 2010

- Der alternde Bewegungsapparat, 2011

- Die Wirbelsäule der Frau, 2012

- OP or not OP - Erkrankungen des Bewegungsapparates in der Praxis, 2013

- Das Zervikalsyndrom mit seinen "Facetten", 2014

- Die gestörte Wirbelsäule - eine Aufgabe für die Praxis - vom Befund zur Behandlung, 2015

leisten, die neben den Kursen weitere Ausbildungsmöglichkeiten anbieten (- Infobox 5).

Zielgruppen sind vor allem die Ärzte für Allgemeinmedizin, welche in Österreich keine Ausbildung in konservativer Orthopädie erhalten, obwohl am Bewegungsapparat erkrankte Patienten einen Großteil ihres Patientenklientels stellen.

Durch die für uns bedauerlich geringe Kontaktpflege mit unserer fachlichen Standesvertretung ist uns nicht bekannt, wo niedergelassene Orthopäden ihre
Ausbildung für die medizinische Problematik von Patienten erhalten haben, die im niedergelassenen Bereich vorstellig werden und mit ihren Problemen häufig deutlich von den im stationären Bereich zu beobachtenden Erkrankten differieren. Im Vordergrund steht hier das Lernen von klinischen Untersuchungstechniken, die bekannterweise unbedingte Voraussetzung für alle therapeutischen Maßnahmen darstellen.

Erklärbar wären diese Umstände zum einen durch die Größe des Gebiets der chirurgischen Orthopädie und Unfallchirurgie, die bei der Verfolgung ihrer Ziele im Laufe der Zeit chirurgisches Denken entwickelt hat - nämlich das Denken in der Pathomorphologie - und zum anderen damit, dass ein Wechsel in konservativ-orthopädische Sichtweisen im Sinne der neuromuskuloskelettalen Medizin nicht leicht scheint, was auch ausbildungspolitische Konzepte sehr erschwert. Im vorliegenden Fall wäre eine Kompetenzverteilung notwendig, nämlich eine Leitung der konservativen Orthopädie, die imstande ist, die drei Sparten der Orthopädie zusammenzuhalten.

Zu bemerken wären auch Entwicklungen in Österreich, die nicht unbedingt unter dem Einfluss der Medizin stehen:

- Steigende Tendenzen, Menschen im stationären Bereich zu behandeln,

- Tendenzen, stationär aufgenommene Patienten operativen Eingriffen zu unterziehen,

- Tendenzen, konservative Behandlungen unterzubewerten,

- Tendenzen der Einflussnahme von Nichtmedizinern auf die Medizin,

- Tendenzen, Nichtmediziner in vermehrtem Maße in ärztliche Betreuungsmaßnahmen zu integrieren.

Der konsequenterweise erfolgenden geringeren Bewertung der konservativ-orthopädischen Möglichkeiten müsste mit einer (internationalen?) vorerst deutschsprachigen Schule begegnet werden.

\section{Korrespondenzadresse}

Univ.-Prof. Dr. H. Tilscher

Öst. Ärztegesellschaft für Manuelle Medizin, Geriatriezentrum am Wienerwald Jagdschlossgasse 59, 1130 Wien, Österreich hans.tilscher@extern.wienkav.at

Dr. A. Lechner

Schweizertalstraße 4/4/9, 1130, Wien Österreich

alexander-lechner@chello.at

Interessenkonflikt. H. Tilscher und A. Lechner geben an, dass kein Interessenkonflikt besteht. 